\title{
Probing interfacial energetics and charge transfer kinetics in semiconductor nanocomposites: New insights into heterostructured $\mathrm{TiO}_{2} / \mathrm{BiVO}_{4}$ photoanodes
}

Lucas H. Hess, Jason K. Cooper, Anna Loiudice, Chang-Ming Jiang, R. Buonsanti*, and Ian D. Sharp*

Dr. L.H. Hess, Dr. J.K. Cooper, Dr. C.-M. Jiang, Dr. I.D. Sharp

Joint Center for Artificial Photosynthesis and Chemical Sciences Division, Lawrence Berkeley National Laboratory, One Cyclotron Rd., Berkeley, CA 94720, United States

*Email: idsharp@lbl.gov

Dr. A. Loiudice, Prof. R. Buonsanti

Department of Chemical Sciences and Engineering, École Polytechnique Fédérale de Lausanne, Sion, CH-1950, Switzerland

*Email: raffaella.buonsanti@epfl.ch

Keywords: nanocomposites, photoelectrochemistry, interfacial energetics, photocarrier dynamics, metal oxides

\begin{abstract}
Heterostructured nanocomposites offer promise for creating systems exhibiting functional properties that exceed those of the isolated components. For solar energy conversion, such combinations of semiconducting nanomaterials can be used to direct charge transfer along pathways that reduce recombination and promote efficient charge extraction. However, interfacial energetics and associated kinetic pathways often differ significantly from predictions derived from the characteristics of pure component materials, particularly at the nanoscale. Here, the emergent properties of $\mathrm{TiO}_{2} / \mathrm{BiVO}_{4}$ nanocomposite photoanodes are explored using a combination of X-ray and optical spectroscopies, together with photoelectrochemical (PEC) characterization. Application of these methods to both the pure components and the fully assembled nanocomposites reveals unpredicted interfacial energetic alignment, which promotes ultrafast injection of electrons from $\mathrm{BiVO}_{4}$ into $\mathrm{TiO}_{2}$. Physical charge separation yields extremely long-lived photoexcited states and correspondingly enhanced photoelectrochemical functionality. This work highlights the importance of probing
\end{abstract}


emergent interfacial energetic alignment and kinetic processes for understanding mechanisms of solar energy conversion in complex nanocomposites.

\section{Introduction}

Photoelectrochemical (PEC) systems, in which solar energy is captured and converted into stored energy in chemical bonds, offer promise for production of renewable and sustainable fuels.[1, 2] Such artificial photosystems are investigated for the generation of hydrogen via water reduction or carbon-based fuels via carbon dioxide reduction. These reduction reactions often occur at p-type semiconductor photocathodes, a variety of which are actively investigated.[3,4] Regardless of the desired product, efficient and stable photoanodes are required to drive the water oxidation half reaction. These $\mathrm{n}$-type semiconductors must possess bandgaps that are suitable for harvesting solar radiation, band edge positions that support the generation of large photovoltages, and (photo)chemical stability under strongly oxidative conditions. In addition, photogenerated charge carriers must be efficiently separated in these materials and transported across their interfaces. At present, no single material possesses a suitable combination of properties to meet all of these requirements.[3] To overcome this critical materials gap, nanocomposite photoanode architectures are particularly attractive since they provide the opportunity to assemble different materials with combinations of desired properties into heterostructures with appropriate length scales to achieve improved functionality. Despite this promise, the internal mechanisms governing energetics and kinetics of charge separation and extraction in complex nanostructured materials are frequently unknown. Indeed, predictions of behavior derived from pure compound properties cannot account for emergent phenomena that govern function in many mixed phase nanosystems. In this work, we use a combination of X-ray and optical spectroscopic methods, together with PEC measurements, to probe fundamental charge transport and recombination pathways in heterostructured nanocomposites of titanium dioxide and bismuth vanadate $\left(\mathrm{TiO}_{2} / \mathrm{BiVO}_{4}\right)$. Our approach is to directly probe optoelectronic processes in fully assembled systems, which 
reveals significant deviations from predictions based on measurements of the individual material constituents. In addition to resolving conflicting mechanistic assignments of function in $\mathrm{TiO}_{2} / \mathrm{BiVO}_{4}$ nanocomposites, this work establishes a general methodology for understanding charge transport and recombination pathways in complex semiconductor nanosystems. Knowledge of such mechanisms will be critical for the rational design of a next generation of heterostructured nanocomposites for improved solar energy utilization.

Among semiconductors investigated as photoanodes, transition metal oxides have attracted considerable interest due to their potential for improved stability relative to main group semiconductors under oxidative conditions. Monoclinic scheelite $\mathrm{BiVO}_{4}$ is a prototypical example of a transition metal oxide that possesses both desirable and undesirable properties for PEC energy conversion.[3, 5] It has a moderate bandgap of $2.5 \mathrm{eV}$ and an energetically low-lying valence band position.[6, 7] However, it suffers from exceptionally small majority carrier mobilities due to formation of electron small polarons,[8-10] photochemical instabilities associated with localization of holes near its surface,[11] and relatively slow injection of holes across the semiconductor/electrolyte interface. While incorporation of a catalyst on the surface can improve stability and increase OER activity, reducing photocarrier recombination in the material calls for strategies to promote physical charge separation and electron extraction.

In recent years, significant progress has been made in improving the PEC performance characteristics of $\mathrm{BiVO}_{4}$ by interfacing it with larger bandgap metal oxides possessing favorable electron transport properties, typically in nanostructured composites. Most notably, $\mathrm{BiVO}_{4} / \mathrm{WO}_{3}$ heterostructures have yielded large photocurrent densities, above $5.3 \mathrm{~mA} \mathrm{~cm}{ }^{-2}$ at $1.23 \mathrm{~V}$ vs. the reversible hydrogen electrode (RHE),[12] which approach the theoretical saturation current density limit of $6.2 \mathrm{~mA} \mathrm{~cm}^{-2}$ for a $2.5 \mathrm{eV}$ bandgap semiconductor. This improvement is expected based on the known band positions of the two materials, which allow for selective injection of electrons from the conduction band (CB) of $\mathrm{BiVO}_{4}$ into that of 
$\mathrm{WO}_{3}$. In appropriately designed nanocomposites, electron injection can occur in the ps time regime, which suppresses recombination and enables photo-generated holes in $\mathrm{BiVO}_{4}$ to persist for times on the order of a second.[13]

While photocurrent densities achieved with $\mathrm{WO}_{3} / \mathrm{BiVO}_{4}$ systems are impressive, the $\mathrm{pH}$ stability windows for $\mathrm{WO}_{3}$ and $\mathrm{BiVO}_{4}$ are not well matched, which could introduce additional degradation pathways, and $\mathrm{WO}_{3}$ is characterized by a very positive flatband potential, which could reduce the maximum achievable photovoltage from such assemblies.[14] As an alternative approach, a variety of studies have investigated nanocomposite heterostructures between $\mathrm{BiVO}_{4}$ and $\mathrm{TiO}_{2}$.[14-23] Such a system represents an interesting case in which basic predictions deviate significantly from functional characteristics. In particular, established band edge positions for pure $\mathrm{BiVO}_{4}$ and pure $\mathrm{TiO}_{2}$ should yield Type I band alignment, which would prevent electron injection from $\mathrm{BiVO}_{4}$ into $\mathrm{TiO}_{2}$. The predicted result would be accumulation of photogenerated electrons and holes on the $\mathrm{BiVO}_{4}$ side of the interface and reduced PEC performance. In stark contrast to this prediction, studies have frequently observed enhanced PEC activity in $\mathrm{TiO}_{2} / \mathrm{BiVO}_{4}$ heterostructures relative to the pure phase components.[14-23] The origin of this enhancement has been variously attributed to hot electron injection from $\mathrm{BiVO}_{4}$ to $\mathrm{TiO}_{2},[20]$ an unidentified defect-mediated charge transport pathway,[15] and the existence of unexpected Type II band alignment instead of the predicted Type I alignment.[14, 16-19] For example, Cheng and co-workers recently provided experimental evidence for Type II alignment in planar heterostructures between $\mathrm{BiVO}_{4}$ and rutile $\mathrm{TiO}_{2}$.[23] Meanwhile, other experimental studies have concluded that Type I band alignment is present and electron injection from $\mathrm{BiVO}_{4}$ to $\mathrm{TiO}_{2}$ does not occur.[21, 22] However, limited evidence for these mechanisms was provided in each of these studies and conclusions were often based on assumptions regarding properties of the isolated pure phase materials. Despite the lack of consistent experimental conclusions and the discrepancy between prediction and observation, direct measurement of junction energetics and charge 
transfer kinetics in fully assembled composites remains lacking.[24] Understanding the surprising behavior that emerges in $\mathrm{TiO}_{2} / \mathrm{BiVO}_{4}$ heterostructures is important for maximizing performance in this system and, more broadly, for identifying emergent phenomena in heterostructured nanocomposites that are expected to play a central role in meeting the demanding requirements associated with PEC energy conversion.

\section{Materials and methods}

Materials: Bismuth (III) chloride (98\%) ( $\left.\mathrm{BiCl}_{3}\right)$, vanadyl acetylacetonate $(99.99 \%)$ $\left(\mathrm{VO}(\mathrm{acac})_{2}\right)$, titanium tetrachloride, 1-octadecene (technical grade, 90\%), oleic acid (technical grade, 90\%) (OLAC) and oleylamine (technical grade, 70\%) (OLAM) were purchased from Aldrich and used without further purification.

$\mathrm{Bi}_{2} \mathrm{O}_{2.7} / \mathrm{VO}_{x}$ synthesis: $3.17 \mathrm{mmol}$ of $\mathrm{BiCl}_{3}$ was dispersed in $10 \mathrm{~mL}$ of OLAM in a $25 \mathrm{~mL}$ three-necked flask equipped with a condenser and a thermocouple held by a glass tube. The solution was continuously stirred and kept under vacuum for $1 \mathrm{~h}$. The solution was then heated to $170{ }^{\circ} \mathrm{C}$ in the course of 15 min and then kept at that temperature for $1 \mathrm{~h}$. During this entire time, it was necessary to keep a flow of $\mathrm{N}_{2}$ through the flask in order to obtain best results. After this time, the color of the $\mathrm{BiCl}_{3}$ solution turned from milky white to dark gray. A solution of $1.27 \mathrm{mmol}$ of $\mathrm{VO}(\mathrm{acac})_{2}$ in $1 \mathrm{~mL}$ of OLAM was then swiftly injected into the flask. The solution turned black after injection and the nanocrystals were allowed to grow for $30 \mathrm{~min}$ at $250{ }^{\circ} \mathrm{C}$. The reaction was then quenched with cold hexane. Unsolubilized materials were removed by centrifugation, and ethanol was added dropwise to the supernatant until it became turbid. The mixture was centrifuged, the supernatant discarded, and the precipitated nanoparticles redispersed in hexane.

$\mathrm{TiO}_{2}$ branched nanorods synthesis: $\mathrm{TiO}_{2}$ NRs were obtained following the procedure reported by R. Buonsanti et al.,[25] $3 \mathrm{~g}$ of 1-octadecene, $3 \mathrm{mmol}$ of OLAM and $11 \mathrm{mmol}$ of OLAC were loaded in a three-neck flask and degassed at $120{ }^{\circ} \mathrm{C}$ for $45 \mathrm{~min}$, after which the mixture was cooled down to $50{ }^{\circ} \mathrm{C}$ under $\mathrm{N}_{2}$ flow. At this point, $1 \mathrm{mmol}$ of titanium tetrachloride 
dissolved in $1 \mathrm{~mL}$ of 1 - octadecene was added and the flask was heated up to $290{ }^{\circ} \mathrm{C}$ at a ramp rate of $25^{\circ} \mathrm{C} \min ^{-1}$. After heating for $1 \mathrm{~h}$ at $290{ }^{\circ} \mathrm{C}$, the reaction was stopped.

Nanocomposite electrode preparation: First, different nanocomposite mixtures were prepared by mixing the $\mathrm{TiO}_{2} \mathrm{NRs}$ or $\mathrm{Al}_{2} \mathrm{O}_{3} \mathrm{NCs}$ (purchased from Aldrich (20 wt.\% in isopropanol)) with $\mathrm{Bi}_{2} \mathrm{O}_{2.7} / \mathrm{VO}_{\mathrm{x}}$ heterodimers (both in hexane solution) in a weight ratio of $1: 1$. The $\mathrm{Al}_{2} \mathrm{O}_{3}$ NCs were removed from the original solvent by centrifugation and re-dispersed in hexane, resulting in well-dispersed NCs of around $50 \mathrm{~nm}$ in size. The as prepared mixtures were stirred for $2 \mathrm{~h}$ to ensure a good mixing. After that, the electrodes were fabricated on FTO using a layer-by-layer technique. $50 \mu \mathrm{l}$ of nanocomposite suspension was spin-coated at 700 rpm for $45 \mathrm{~s}$. The film was then annealed at $500{ }^{\circ} \mathrm{C}$ for $10 \mathrm{~min}$. The sequence was repeated to achieve a film thickness of $200 \mathrm{~nm}$. Pure $\mathrm{BiVO}_{4}$ and pure $\mathrm{TiO}_{2}$ films were prepared following the same procedure. Excess of $\mathrm{V}_{2} \mathrm{O}_{5}$ present in the $\mathrm{BiVO}_{4}$ electrodes was removed by soaking them in $1 \mathrm{M} \mathrm{NaOH}$ solution for 30 s with gentle stirring. The resulting electrodes were rinsed with DI water and dried.

Photoelectrochemical measurements: Photoelectrochemical performance characteristics of photoanodes were evaluated in a typical undivided three-electrode configuration using a $\mathrm{CHI}$ 604D Potentiostat/Galvanostat. The light source was an AM 1.5 solar simulator (Solar Light Co, Inc. model XPS 300) with the illumination intensity adjusted to $100 \mathrm{~mW} / \mathrm{cm}^{2}$. All illuminated areas were approximately $0.2 \mathrm{~cm}^{2}$. Photocurrent measurements were performed in a $1 \mathrm{M}$ potassium phosphate buffer solution ( $\mathrm{pH}$ 6.8) with $0.1 \mathrm{M}$ sodium sulfite $\left(\mathrm{Na}_{2} \mathrm{SO}_{3}\right)$ as a hole scavenger. Photocurrents were monitored while sweeping the potential to the positive (anodic) direction with a scan rate of $10 \mathrm{mV} / \mathrm{s}$. A set of 5 photoelectrodes was tested for each studied structure. While all measurements were carried out using a $\mathrm{Ag} / \mathrm{AgCl}(4 \mathrm{M} \mathrm{KCl})$ reference electrode, all results in this work are presented against the reversible hydrogen electrode (RHE) for ease comparison with other papers that use electrolytes with different $\mathrm{pH}$ 
conditions. The conversion between potentials vs. $\mathrm{Ag} / \mathrm{AgCl}$ and vs. RHE is performed using the following equation:

$$
E(v s . \mathrm{RHE})=E(\text { vs. } \mathrm{Ag} / \mathrm{AgCl})+E_{\mathrm{Ag} / \mathrm{AgCl}}(\text { reference })+0.0591 V \times p \mathrm{H}
$$

with $E_{\mathrm{Ag} / \mathrm{AgCl}}($ reference $)=0.1976 \mathrm{~V}$ vs. $\mathrm{NHE}$ at $25^{\circ} \mathrm{C}$

Incident photon-to-charge conversion efficiency (IPCE) was measured using an home built setup. A Newport 150W Xe lamp and an Oriel Cornerstone 1/8 m monochromator with 1.5 mm slits, yielding a spectral resolution of better than $0.5 \mathrm{~nm}$ was used as a light source. A beam splitter, together with a Thorlabs FDS1010-CAL calibrated Si photodiode, was used to monitor the intensity of the monochromatic output during the measurement. In order to ensure accurate measurement, the incident light spot under-filled both the sample and reference diode. A Gamry Reference 600 potentiostat was used in combination with a three-electrode electrochemical cell to maintain the sample at the reported potentials and to measure the current of the photoanode. The IPCE was then calculated based on the following formula:

$$
\operatorname{IPCE}(\%)=\frac{1240 \times J_{p h}}{I_{i} \times \lambda} \times 100
$$

where $J_{p h}$ is the measured photocurrent density in $\mathrm{mA} / \mathrm{cm}^{2}, I_{i}$ is the incident light intensity in $\mathrm{mW} / \mathrm{cm}^{2}$, and $\lambda$ is the incident photon wavelength in $\mathrm{nm}$.

Trasmission electron microscopy (TEM): TEM was carried out on JEOL 2100F microscope operating at $200 \mathrm{kV}$. Samples were prepared by drying a drop of hexane solution containing the NCs on the surface of a carbon-coated copper grid (Ted Pella, Inc.) or by gently scratching the nanocomposite film with the grid.

Wide-angle XRD: XRD was performed using a Bruker D8-Advanced X-ray diffractometer equipped with a GADDS area detector operating at $\mathrm{Cu}-\mathrm{K} \alpha$ wavelength of $\lambda=1.54 \AA$. Samples were prepared by drop casting NCs on silicon substrates.

X-ray photoelectron spectroscopy (XPS): XPS was performed using a monochromatized Al $\mathrm{K} \alpha$ source $(\mathrm{h} v=1486.6 \mathrm{eV})$, operated at $15 \mathrm{~W}$, on a Kratos Axis Ultra DLD system at a 
takeoff angle of $0^{\circ}$ relative to the surface normal and a pass energy for narrow scan spectra of $20 \mathrm{eV}$, corresponding to an instrument resolution of approximately $600 \mathrm{meV}$.

Transient absorption spectroscopy (TAS): Measurements were conducted on thin films formed on quartz substrates. The pump beam was produced by a Coherent Libra (Coherent, CA, USA) laser coupled to a Coherent OPerA Solo optical parametric amplifier (OPA). The output was directed to the sample as the excitation pump, with a wavelength of $350 \mathrm{~nm}$, pulse duration of $150 \mathrm{fs}$, repetition rate of $1 \mathrm{kHz}$, pulse energy of $700 \mathrm{~nJ}$, and beam diameter of 0.3 $\mathrm{mm}$ at the sample. For measurements in the sub-ns time regime, the white light probe pulse was produced by directing a portion of the $800 \mathrm{~nm}$ fundamental output from the Ti:sapphire laser through a delay stage and onto a $\mathrm{CaF}_{2}$ crystal, which was continuously translated to prevent beam damage. The transient absorption measurement system was produced by Ultrafast Systems.

For TAS measurements in the ms-s time regime, a custom setup was used. The samples were

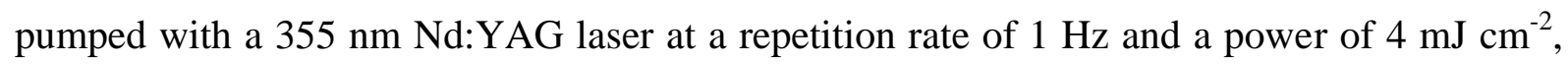
generated by a Minilite II system (Continuum, USA). For probe light was produced by a quartz tungsten halogen lamp (250W, Newport Corporation, USA) and the wavelength was selected with a CS260 monochromator (Newport Corporation, USA). The light transmitted through the sample was detected by a Si avalanche photodetector (APD120A2, Thorlabs Inc., USA). To filter unwanted stray light, the photodiode was mounted at the exit of a MS257 monochromator (Newport Corporation, USA) set to the same wavelength as the monochrometer. The diode signal was amplified by a low noise voltage preamplifier (SR560, Stanford Research Systems Inc., USA) and recorded with a DPO4054 oscilloscope (Tektronix Inc., USA) triggered with respect to the laser pulses. A total of 2500 transients were recorded and averaged for each measurement. Finally, the data was averaged logarithmically in time.

Photoluminescence (PL): PL measurements were performed on a custom-built setup. Temperature control was achieved using a $10 \mathrm{~K}$ closed cycle He cryostat (Janus Research 
Company, Wilmington, MA, USA) equipped with a temperature controller (Model 335, Lake Shore Cryotronics, Inc., Westerville, OH, USA). PL spectra were recorded using an Andor spectrometer equipped with a 600 lines $\mathrm{mm}^{-1}$ grating with $500 \mathrm{~nm}$ blaze under $340 \mathrm{~nm}$ excitation using a Coherent OPerA Solo optical parametric amplifier pumped with a Coherent Libra amplified Ti:S system. Wavelength calibration was performed with a $\mathrm{Hg}$ calibration lamp and correction for the spectral response of the setup was achieved using a 45 W NISTtraceable quartz tungsten halogen light source with known color temperature.

\section{Results and Discussion}

\subsection{Formation of Heterostructured $\mathrm{TiO}_{2} / \mathrm{BiVO}_{4}$ Nanocomposite Films}

Recently, we reported a versatile approach to formation of multicomponent oxides, as well as heterostructured nanocomposites composed of different metal oxide semiconductors.[13] Here, we take advantage of the synthetic flexibility afforded by this method to fabricate and characterize nanocomposite films comprising monoclinic $\mathrm{BiVO}_{4}$ nanocrystals (NCs) and anatase $\mathrm{TiO}_{2}$ branched nanorods (NRs). Complete synthesis details are provided in the Supporting Information. In brief, $\mathrm{Bi}_{2} \mathrm{O}_{2.7} / \mathrm{VO}_{\mathrm{x}} \mathrm{NC}$ heterodimers, approximately $20 \mathrm{~nm}$ in size

(Figure 1a), were formed using a seed-mediated colloidal growth process. Hexane solutions containing a 50/50 weight ratio of these heterodimers and $\mathrm{TiO}_{2} \mathrm{NRs}$, which were approximately $30-50 \times 120 \mathrm{~nm}$ in size (Figure 1b), were spin-coated onto desired substrates. After annealing the $\mathrm{Bi}_{2} \mathrm{O}_{2.7} / \mathrm{VO}_{\mathrm{x}}$ heterodimer/ $/ \mathrm{TiO}_{2}$ nanocomposite film in air at $500{ }^{\circ} \mathrm{C}$ for only 10 min, monoclinic $\mathrm{BiVO}_{4}$ was formed and the $\mathrm{TiO}_{2} \mathrm{NR}$ morphology was preserved. Figure 1c shows a representative transmission electron micrograph (TEM) of the resulting $\mathrm{TiO}_{2} / \mathrm{BiVO}_{4}$ nanocomposites, showing that there is an intimate contact between the $\mathrm{TiO}_{2}$ and $\mathrm{BiVO}_{4}$ domains. For reference purposes, pure films of $\mathrm{BiVO}_{4}$ and of $\mathrm{TiO}_{2} \mathrm{NRs}$ were also synthesized. X-ray diffraction (XRD) data, shown in Figure 1d, confirm the presence of monoclinic scheelite $\mathrm{BiVO}_{4}$ and anatase $\mathrm{TiO}_{2}$ in each of the corresponding reference films 
and of only these two phases in the heterostructured nanocomposite. No apparent peak shift of the monoclinic $\mathrm{BiVO}_{4}$, which would indicate Ti-bulk alloying, was observed in the XRD pattern of the composite. While this does not eliminate the possibility of low concentration Ti incorporation in $\mathrm{BiVO}_{4}$, previous work by Bard and co-workers found no significant change of PEC characteristics from Ti-incorporated $\mathrm{BiVO}_{4}$, suggesting that $\mathrm{Ti}$ does not act as an electronically active dopant in $\mathrm{BiVO}_{4}$.[26] The use of pre-crystallized building blocks allows for much shorter annealing times compared to other methods, which preserves the nanoscale dimensions of both components with minimal ripening or interdiffusion. For comparative studies of interfacial charge transfer processes, heterostructured nanocomposite films composed of $\mathrm{BiVO}_{4}$ and $\mathrm{Al}_{2} \mathrm{O}_{3} \mathrm{NCs}$ were also fabricated.

\subsection{Photoelectrochemical Performance Characteristics}

The PEC performance characteristics of nanocomposite film, along with the pure component films, deposited on degenerately-doped $\mathrm{p}^{++} \mathrm{Si}$ were evaluated by measuring the photocurrent density as a function of applied electrochemical potential $(J-E)$, as shown in Figure 2a. A comparison of illuminated and dark $J-E$ curves is provided in the Supporting Information (Figure S1). Measurements were conducted under simulated AM1.5G solar irradiation at $100 \mathrm{~mW} \mathrm{~cm}$ and in the presence of $0.1 \mathrm{M} \mathrm{Na}_{2} \mathrm{SO}_{3}$ as a hole scavenger. The presence of a hole scavenger in the solution enables determination of the inherent performance of these nanocomposites without convoluting the potentially variable catalytic activities of their surfaces or needing to incorporate a separate water oxidation catalyst, which is beyond the scope of this work. Comparison of the $J-E$ characteristic from a pure phase $\mathrm{BiVO}_{4}$ film with that of the mixed $\mathrm{TiO}_{2} / \mathrm{BiVO}_{4}$ nanocomposite film reveals an approximate doubling of the photocurrent in the heterostructured nanocomposite at $1.23 \mathrm{~V}$ vs. RHE, while the pure $\mathrm{TiO}_{2}$ film exhibited comparatively negligible photocurrent. The onset potential for photoanodic current from the $\mathrm{TiO}_{2} / \mathrm{BiVO}_{4}$ nanocomposites was very close to that of the pure 
$\mathrm{BiVO}_{4}$ film, suggesting that photoholes are mainly transferred from the $\mathrm{BiVO}_{4}$ valence band to the sulfite. However, while the $\mathrm{TiO}_{2} / \mathrm{BiVO}_{4}$ nanocomposite shows significantly enhanced photocurrent densities at more anodic potentials, its performance at low potentials $(<0.8 \mathrm{~V}$ vs. RHE) is comparable to the pure $\mathrm{BiVO}_{4}$ film. This behavior suggests that photocarrier recombination at $\mathrm{BiVO}_{4} / \mathrm{TiO}_{2}$ interfaces is significant, but is suppressed by the enhanced driving force for photocarrier separation at higher potentials. Such a behavior is commonly observed in nanostructured photoanodes [27] and is also consistent with the observation of radiative recombination at interfaces obtained from PL results discussed below. These results are consistent with previous findings from $\mathrm{TiO}_{2} / \mathrm{BiVO}_{4}$ heterostructures, in which $\mathrm{PEC}$ performance characteristics have been observed to improve in composites, despite the unfavorable band alignment predicted by considering the energetics of each semiconductor in isolation.[28-33] The photocurrents obtained here are lower than the highest reported for $\mathrm{BiVO}_{4}$ by a factor of approximately four.[34] While $\mathrm{BiVO}_{4}$ is one of the best-performing metal oxide photoanode materials,[3] it is important to note that the present work is devoted to foundational understanding of interfacial energetics and charge transfer processes and we have not attempted to optimize film thickness, phase ratio, or component length scales. Given the modular and tunable nature of this synthetic route to heterostructured nanocomposites, there is significant future opportunity for improving function.

In agreement with the $J-E$ characteristics, the incident-photon-to-charge efficiency (IPCE), measured at an applied electrochemical bias of $1.23 \mathrm{~V} v s$. RHE, was also observed to approximately double in the $\mathrm{TiO}_{2} / \mathrm{BiVO}_{4}$ nanocomposites, with a maximum IPCE of $30 \%$ at a wavelength of $430 \mathrm{~nm}$ (Figure 2b). Despite changes of efficiency, the spectral shape of the IPCE curves obtained from pure $\mathrm{BiVO}_{4}$ and from $\mathrm{TiO}_{2} / \mathrm{BiVO}_{4}$ nanocomposites are nearly identical. Based on this finding, the previously suggested hot carrier model for electron injection from the $\mathrm{CB}$ of $\mathrm{BiVO}_{4}$ to the $\mathrm{CB}$ of $\mathrm{TiO}_{2}$ with Type I band alignment,[20] is highly unlikely. In such a scenario, the charge transfer would only occur if photogenerated electrons 
were excited to higher energies in the $\mathrm{CB}$ of $\mathrm{BiVO}_{4}$ and were to transfer before relaxation to the $\mathrm{CB}$ edge. Due to the additional energy required to overcome such an energy barrier at the interface, it would be expected that a hot carrier-based mechanism would lead to an additional step-like increase of IPCE at shorter wavelengths, which is not observed experimentally. Furthermore, we note that for such a Type I band alignment, back electron transfer from $\mathrm{TiO}_{2}$ to $\mathrm{BiVO}_{4}$ would be energetically favored and would compete with electron extraction to the underlying electrical contact. Together, the $J-E$ and IPCE characteristics suggest Type II band alignment in the nanocomposite film, in contrast to initial expectations based on literature values for the electron affinities of the two materials.

\subsection{Interface Energetics in Nanoscale Composites}

In order to understand the mechanism of PEC performance enhancements in the $\mathrm{TiO}_{2} / \mathrm{BiVO}_{4}$ nanocomposites, we performed X-ray photoelectron spectroscopy (XPS) and contact potential difference (CPD) measurements. These measurements were first applied to the pure phase reference films in order to provide a basis for comparing traditional predictions to direct measurements of $\mathrm{TiO}_{2} / \mathrm{BiVO}_{4}$ interface energetics in nanocomposites. To this end, $\mathrm{BiVO}_{4} \mathrm{NC}$ and $\mathrm{TiO}_{2} \mathrm{NR}$ films were prepared on highly doped $\mathrm{p}^{++} \mathrm{Si}$ substrates. Figure 3a shows valence band spectra obtained from these films, along with the reference spectrum of a clean $\mathrm{p}^{++} \mathrm{Si}$ sample. The measured spectra yield valence band edges of $1.9 \mathrm{eV}$ and $2.2 \mathrm{eV}$ below the Fermi level for the $\mathrm{BiVO}_{4}$ and $\mathrm{TiO}_{2}$ films, respectively. Given the well-known band gaps of $\mathrm{BiVO}_{4}(2.5 \mathrm{eV})$ and anatase $\mathrm{TiO}_{2}(3.2 \mathrm{eV})$, these results indicate that the assynthesized semiconductor films are n-type, with Fermi levels positioned at $0.6 \mathrm{eV}$ and $1.0 \mathrm{eV}$ below the conduction band edges, respectively. While the materials were not intentionally doped, this n-type character is likely due to the presence of oxygen vacancy defects in both materials and unintentionally incorporated hydrogen may also contribute to doping of the $\mathrm{BiVO}_{4} \cdot[35-37]$ To predict the relative energy level alignment between the two oxides, the work functions were determined with respect to a $\mathrm{Au}$ probe via CPD. Calibration of work 
function values was achieved by measuring the CPD of the Au probe relative to a freshlycleaved highly oriented pyrolytic graphite (HOPG) sample, whose work function was taken to be $4.5 \mathrm{eV}$ in accordance with prior literature.[38] Work functions of $4.4 \mathrm{eV}$ and $4.2 \mathrm{eV}$, which imply electron affinities of $4.0 \mathrm{eV}$ and $3.8 \mathrm{eV}$, were measured for the $\mathrm{BiVO}_{4}$ and $\mathrm{TiO}_{2}$ samples, respectively. While these electron affinity values are smaller than anticipated based on previous studies, we note that surface energetics are strongly sensitive to the presence of adsorbates, surface termination-induced dipoles, and exposed facets. This leads to a broad range of measured values; the electron affinity of $\mathrm{BiVO}_{4}$ has been reported to lie in the range of $4.4-4.8 \mathrm{eV},[28-30]$ whereas that of anatase $\mathrm{TiO}_{2}$ has been reported to lie in the range of $4.0-5.1 \mathrm{eV} .[31-33]$ Nevertheless, the difference of electron affinity values between the two materials measured here is in agreement with most prior reports, from which a Type I band alignment at their interface would be predicted. However, such an energetic alignment would be expected to reduce PEC performance and cannot account for the enhanced $J-E$ and IPCE characteristics shown in Figure 2.

Considering that both the $\mathrm{BiVO}_{4}$ and $\mathrm{TiO}_{2}$ used in this study are moderately n-type, depletion widths associated with heterojunction formation would be significantly larger than the nanoparticle dimensions. Furthermore, annealing of the assembled heterostructured nanocomposites is expected to strongly modify the interfacial properties. As such, it is not possible to accurately describe the band alignment from measurement of the pure materials in isolation. Nevertheless, we note that ideal heterojunctions between bulk equivalents of these materials should be characterized by downward (upward) band bending on the $\mathrm{BiVO}_{4}\left(\mathrm{TiO}_{2}\right)$ side of the interface. As a consequence, barriers to both electron and hole injection from $\mathrm{BiVO}_{4}$ to $\mathrm{TiO}_{2}$ would be expected.

Since this predicted band alignment is not consistent with functional PEC characteristics of the heterostructured materials, we sought to investigate interfacial charge transfer in the assembled nanocomposites. To this end XPS was performed on $\mathrm{TiO}_{2} / \mathrm{BiVO}_{4}$ nanocomposite 
films in darkness and under photoexcitation. Figure 3b,c shows Ti $2 \mathrm{p}$ and Bi $4 \mathrm{f}$ core level spectra obtained from pure phase materials compared to the nanocomposite assemblies. Spectra of the pure nanoparticle films are consistent with the presence of dominant $\mathrm{Bi}^{3+}$ (Figure 3c) and $\mathrm{V}^{5+}$ (Figure S2) in $\mathrm{BiVO}_{4}$ and $\mathrm{Ti}^{4+}$ (Figure 3b) in $\mathrm{TiO}_{2}$, as expected. Core level peak shapes are preserved in the composite, suggesting no significant local oxidation state changes in either of the components. However, significant shifts of the binding energies are observed for the heterostructured nanocomposite film. Compared to the pure $\mathrm{BiVO}_{4}$ film, both the $\mathrm{Bi} 4 \mathrm{f}$ and $\mathrm{V} 2 \mathrm{p}$ core levels were shifted by approximately $+0.40 \mathrm{eV}$ in the nanocomposite. At the same time, an opposite shift of $-0.34 \mathrm{eV}$ was observed for the Ti $2 \mathrm{p}$ core level compared to the pure $\mathrm{TiO}_{2}$ nanoparticle film. These data allow for direct determination of the interfacial band alignment in the heterostructured nanocomposite film. In particular, the valence band offset, $\Delta E_{v}$, can be calculated according to:[31, 39-42]

$$
\Delta E_{v}=\Delta E_{B}+\left(E_{\mathrm{Ti} 2 p_{3 / 2}}^{\mathrm{TiO}_{2}}-E_{v}^{\mathrm{TiO}_{2}}\right)-\left(E_{\mathrm{Bi}^{2} f_{7 / 2}}^{\mathrm{BiVO}_{7}}-E_{v}^{\mathrm{BiVO}_{4}}\right)
$$

where the $\left(E_{\mathrm{Ti} 2 p_{3 / 2}}^{\mathrm{TiO}_{2}}-E_{v}^{\mathrm{TiO}_{2}}\right)$ is the difference between the Ti $2 p_{3 / 2}$ core level binding energy and the valence band onset in the pure phase $\mathrm{TiO}_{2}$ film and $\left(E_{\mathrm{Bi} 4 f_{7 / 2}}^{\mathrm{BiVO}_{7}}-E_{v}^{\mathrm{BiVO}_{4}}\right)$ is the difference between the $\mathrm{Bi} 4 f_{7 / 2}$ core level binding energy and the valence band onset in the pure $\mathrm{BiVO}_{4}$ film. The quantity $\Delta E_{B}=\left(E_{\mathrm{Bi} 4 f_{7 / 2}}^{\text {composite }}-E_{\mathrm{Ti} 2 p_{3 / 2}}^{\text {composite }}\right)$ is the binding energy difference between the $\mathrm{Bi} 4 f_{7 / 2}$ core level and the $\mathrm{Ti} 2 p_{3 / 2}$ core level in the heterostructured nanocomposite. Importantly, this analysis only relies on energy differences in individually analyzed samples, so it is independent of the Fermi level position and directly yields the valence band offset in the nanocomposite film.[39-41] We note that analysis using the V $2 p_{3 / 2}$ core level positions, rather than the $\mathrm{Bi} 4 f_{7 / 2}$, provided nearly identical results to within the uncertainty of the measurement. Given the valence band offset, measured by XPS using the nanocomposite film itself, along with the bandgaps of $\mathrm{BiVO}_{4}$ and $\mathrm{TiO}_{2}$, it is possible to 
establish a complete portrait of the interfacial band alignment, as shown in Figure 4. In contrast to the prediction of Type I band alignment derived from pure phase measurements (Figure 4a), we find Type II band alignment with a valence band offset, $\Delta E_{v}$, of $\sim 1.5 \mathrm{eV}$ and a conduction band offset, $\Delta E_{c}$, of $\sim 0.8 \mathrm{eV}$ (Figure $4 \mathrm{~b}$ ).

The measured band alignment reveals that photocarrier separation across the $\mathrm{TiO}_{2} / \mathrm{BiVO}_{4}$ interface is energetically favorable, with accumulation of holes in $\mathrm{BiVO}_{4}$ and electrons in $\mathrm{TiO}_{2}$. This finding is consistent with the observed enhancement in PEC characteristics of nanocomposite films. Importantly, these results can be entirely explained with the natural band alignment, without need to invoke alternate mechanisms. Further confirmation of this energetic alignment was obtained from XPS measurements under illumination with a $405 \mathrm{~nm}$ laser, which drives band-to-band excitation in $\mathrm{BiVO}_{4}$ but not in $\mathrm{TiO}_{2}$. Upon illumination, the Ti $2 \mathrm{p}$ core level shifts by approximately $-0.19 \mathrm{eV}$ relative to the dark measurement, as shown in Figure $3 b$. In contrast, the $\mathrm{Bi} 4 \mathrm{f}$ core levels shift by approximately $+0.15 \mathrm{eV}$ relative to its value in darkness (Figure 3c). These shifts are indicative of electron transfer from $\mathrm{BiVO}_{4}$ to $\mathrm{TiO}_{2}$, in which the reduced (increased) electron density in the $\mathrm{BiVO}_{4}\left(\mathrm{TiO}_{2}\right)$ would decrease (increase) electron screening and increase (decrease) the binding energy under illumination.[43-46] Therefore, these shifts observed under photoexcitation are consistent with the energetic alignment (Figure $4 \mathrm{~b}$ ) and resulting accumulation of positive charge within $\mathrm{BiVO}_{4}$ and negative charge in $\mathrm{TiO}_{2}$.

\subsection{Defects and Photocarrier Recombination}

Photoluminescence (PL) spectroscopy was used to provide additional insight into charge transfer and recombination in $\mathrm{TiO}_{2} / \mathrm{BiVO}_{4}$ nanocomposites. We note that, although analyses of PL intensities are commonly used to draw conclusions regarding charge separation and extraction, significant care must be taken when comparing absolute intensities measured from different films and particle assemblies. To avoid erroneous interpretations, we focus on changes of PL spectral weight and features, rather than absolute intensity changes. PL 
measurements were performed on films of pure $\mathrm{TiO}_{2}$, pure $\mathrm{BiVO}_{4}$, and $\mathrm{TiO}_{2} / \mathrm{BiVO}_{4}$ nanocomposites. Room temperature PL was weak from all films, indicating that photocarrier recombination is dominated by non-radiative transitions. Therefore, all measurements were performed at $13 \mathrm{~K}$.

Figure 5 shows normalized PL spectra obtained using a $340 \mathrm{~nm}(3.65 \mathrm{eV})$ pump beam, which allows band-to-band excitation in both $\mathrm{TiO}_{2}$ and $\mathrm{BiVO}_{4}$. In the pure $\mathrm{TiO}_{2}$ film, three major peaks, centered at approximately $440 \mathrm{~nm}, 500 \mathrm{~nm}$, and $540 \mathrm{~nm}$, are observed (Figure S3). These spectral features are consistent with prior reports of PL from anatase $\mathrm{TiO}_{2}$ and can be assigned to radiative recombination of self-trapped excitons in the bulk (440 nm) and of surface-trapped holes with conduction band electrons (500 nm, $540 \mathrm{~nm})$.[47, 48] Very little spectral intensity is observed near $650 \mathrm{~nm}$, which would be the expected position for radiative recombination of surface-trapped electrons with valence band holes.[47, 48] For the case of the pure $\mathrm{BiVO}_{4}$ film, a broad peak near $700 \mathrm{~nm}$ is observed, which has been assigned to radiative recombination at bulk oxygen vacancy sites.[36]

PL from the $\mathrm{TiO}_{2} / \mathrm{BiVO}_{4}$ nanocomposite film is characterized by broad spectral emission. The spectrum is dominated by emission from surface-trapped electrons $(490-570 \mathrm{~nm})$ and holes $(650 \mathrm{~nm})$ at $\mathrm{TiO}_{2}$ surfaces or interfaces. Importantly, the relative PL intensity from radiative recombination of self-trapped excitons within the $\mathrm{TiO}_{2}$ and from oxygen vacancy defects within the $\mathrm{BiVO}_{4}$ is significantly suppressed relative to recombination at surfaces or interfaces. This finding is consistent with the experimentally-determined band alignment, which favors charge separation in both components of the nanocomposites, with electrons from $\mathrm{BiVO}_{4}$ injected into $\mathrm{TiO}_{2}$ and holes from $\mathrm{TiO}_{2}$ injected into $\mathrm{BiVO}_{4}$. The apparent suppression of PL emission from inside of the nanoscale materials highlights the benefit of creating a heterostructured system to separate charge carriers before recombination can occur. However, the recombination processes associated with trapped electrons and holes at interfaces directly contribute to efficiency loss in the assembled system. Such loss processes, 
which are consistent with the finding of relatively low IPCE values, point to future opportunities for engineering interfaces in order to improve efficiency.

\subsection{Charge Transfer and Recombination Kinetics}

In order to investigate the kinetics of charge separation and recombination, transient absorption (TA) spectroscopy was performed on $\mathrm{BiVO}_{4}$-based nanocomposites and results were compared to pure $\mathrm{BiVO}_{4}$ nanoparticle films. For reference, steady-state UV-Vis absorption spectra are provided in the Supporting Information (Figure S4). We note that no significant TA response was observed from pure $\mathrm{TiO}_{2}$. In addition, an $\mathrm{Al}_{2} \mathrm{O}_{3} / \mathrm{BiVO}_{4}$ nanocomposite control sample, in which no interfacial charge transfer is expected, was measured for comparison and to evaluate the role of interfacial recombination in nanocomposites. Figure 6a shows kinetic TA traces in the ps time range from the $\mathrm{BiVO}_{4}$, $\mathrm{Al}_{2} \mathrm{O}_{3} / \mathrm{BiVO}_{4}$, and $\mathrm{TiO}_{2} / \mathrm{BiVO}_{4}$ films, all deposited on fused silica substrates. Measurements were performed using a $350 \mathrm{~nm}(3.54 \mathrm{eV})$ pump beam, which corresponds to a photon energy above the bandgap of $\mathrm{BiVO}_{4}$ and $\mathrm{TiO}_{2}$, and analyzed at a probe wavelength of $475 \mathrm{~nm}$, where the $\mathrm{BiVO}_{4}$ has a maximum signal intensity. The resulting signal arises from the photoexcited state of the material and its evolution provides insight into relaxation processes that occur at ultrafast time scales. As previously reported by us,[13] the photocarrier relaxation dynamics for the pure $\mathrm{BiVO}_{4}$ nanoparticle film and the $\mathrm{Al}_{2} \mathrm{O}_{3} / \mathrm{BiVO}_{4}$ nanocomposite are nearly identical to one another, with minimal relaxation from the initial photoexcited state after $1 \mathrm{~ns}$ (Figure 6a). This result is consistent with the expectation that $\mathrm{Al}_{2} \mathrm{O}_{3}$ presents a large energy barrier for transfer of both electrons and holes.

The ultrafast dynamics of photoexcited $\mathrm{TiO}_{2} / \mathrm{BiVO}_{4}$ nanocomposites differ significantly from those of the pure $\mathrm{BiVO}_{4}$ and from $\mathrm{Al}_{2} \mathrm{O}_{3} / \mathrm{BiVO}_{4}$ nanocomposites. In particular, an additional fast component, with a time constant of $11 \mathrm{ps,} \mathrm{is} \mathrm{observed} \mathrm{and} \mathrm{accounts} \mathrm{for} \mathrm{a} \mathrm{decay}$ of $35 \%$ of the total TA amplitude within the first 50 ps. The emergence of this additional kinetic channel is similar to prior results from $\mathrm{WO}_{3} / \mathrm{BiVO}_{4}$ nanocomposites, in which electron 
injection from $\mathrm{BiVO}_{4}$ to $\mathrm{WO}_{3}$ occurs in the same time range.[11] As discussed above, previously predicted Type I band alignment at the $\mathrm{TiO}_{2} / \mathrm{BiVO}_{4}$ interface would not favor such a charge transfer process. However, these TA results are consistent with the unpredicted, yet experimentally established, Type II band alignment (Figure 4b), in which electron injection from $\mathrm{BiVO}_{4}$ to $\mathrm{TiO}_{2}$ is energetically favorable.

Efficient charge separation relies on rapid electron extraction, which competes directly with photocarrier recombination. In the present case, the nanoscale domains promote fast charge injection, as indicated by the $11 \mathrm{ps}$ time constant. While this pathway appears to compete favorably with much slower photocarrier recombination channels in $\mathrm{BiVO}_{4}$ and on its surfaces, it only accounts for a 35\% reduction of the TA amplitude. This suggests that electron injection, though fast, is not optimally efficient. From a functional standpoint, this finding is also consistent with the observation of a maximum IPCE below 30\% and defectrelated PL, suggesting that additional engineering of the nanocomposite, such as varying the nanoscale component ratio and/or relative surface to volume ratios, could yield considerable performance improvements. While the specific factors that limit charge injection efficiency are not currently known, we note that it has been shown that electron small polarons, rather than delocalized conduction band electrons, are the dominant majority carriers in n-type $\mathrm{BiVO}_{4} \cdot[8-10]$ Although the time scales associated with polaron formation are not yet well established in this material, it is likely that electron injection across the $\mathrm{TiO}_{2} / \mathrm{BiVO}_{4}$ interface also competes with electron self-trapping. These polarons, which are characterized by electron localization at V lattice sites, possess energetic levels that are relatively deep below the CB edge. As a consequence, the band diagram shown in Figure $4 \mathrm{~b}$ may not capture all relevant energetics and the driving force for electron transfer from $\mathrm{BiVO}_{4}$ to $\mathrm{TiO}_{2}$ may be reduced - or even eliminated - upon polaron formation. While a detailed study of polaron properties is outside the scope of the present work, future progress in advancing such systems will benefit greatly from such information. 
Charge separation via electron transfer across the $\mathrm{TiO}_{2} / \mathrm{BiVO}_{4}$ interface is expected to dramatically reduce electron-hole recombination rates, thereby increasing the lifetimes of photogenerated holes that are responsible for driving oxidation reactions. Such processes should result in the enhanced PEC performance characteristics, as observed for the nanocomposite films. While the TA measurements described above provide insight into the initial charge transfer event, which occurs on ps time scales, understanding the kinetics associated with relaxation of the charge-separated state to the ground state requires probing at much longer time scales. Therefore, we performed an additional set of TA measurements in the ms - s time range, which is also the regime that is most relevant for driving chemical reactions. The evolution of TA amplitudes were probed at a wavelength of $550 \mathrm{~nm}$, which has been previously determined to be in a spectral region dominated by holes in $\mathrm{BiVO}_{4}$.[49] As shown in Figure 6b, nearly identical relaxation kinetics are observed for the pure $\mathrm{BiVO}_{4}$ and the $\mathrm{Al}_{2} \mathrm{O}_{3} / \mathrm{BiVO}_{4}$ nanocomposites, with complete decay to the ground state within $10 \mathrm{~ms}$. By contrast, photohole lifetimes are dramatically longer in $\mathrm{TiO}_{2} / \mathrm{BiVO}_{4}$ nanocomposites, with $\mathrm{TA}$ intensity persisting to beyond $1 \mathrm{~s}$. This result provides further confirmation of effective charge separation in heterostructured nanocomposites, enabled by the unexpected Type II band alignment at interfaces between $\mathrm{BiVO}_{4}$ and $\mathrm{TiO}_{2}$. These longer lifetimes correspond to suppressed photocarrier recombination, which results in a higher probability for photogenerated holes in $\mathrm{BiVO}_{4}$ to cross the semiconductor/electrolyte interface. As a consequence, PEC performance characteristics are significantly improved, as evidenced by the approximate doubling of the photocurrent density and the IPCE in nanocomposite films.

\section{Conclusion}

We have demonstrated that heterostructured $\mathrm{TiO}_{2} / \mathrm{BiVO}_{4}$ nanocomposites exhibit enhanced photoelectrochemical performance relative to the pure phase components and found that this behavior arises from unpredicted interfacial energetic alignment that promotes the formation of long-lived charge separated states. In particular, both the nanocomposite and the pure 
components were investigated by a combination of X-ray and optical spectroscopies in order to understand the basic mechanisms contributing to these functional characteristics. An attempt to characterize interface energetics based on pure phase materials led to an expectation of Type I band alignment, which should inhibit charge separation and reduce PEC surface reactions. In stark contrast, X-ray photoelectron spectroscopy on fully assembled nanocomposites revealed that Type II band alignment arises due to interfacial interactions that could not be predicted from the properties of the individual materials. Photo-induced charge transfer across nanoscopic $\mathrm{TiO}_{2} / \mathrm{BiVO}_{4}$ interfaces was confirmed by the suppression of bulk recombination pathways, as measured by photoluminescence spectroscopy. Furthermore, quantification of photocarrier kinetics via ultrafast transient absorption spectroscopy confirmed that competition between interfacial charge transfer and recombination favors selective electron injection from $\mathrm{BiVO}_{4}$ to $\mathrm{TiO}_{2}$. This physical separation of charge carriers was found to significantly reduce photocarrier recombination rates, with photoexcited holes persisting to times as long as $0.1-1 \mathrm{~s}$, a range that is well matched to the natural time scales for chemical reactions. These results resolve apparent contradictions between a number of prior studies on $\mathrm{TiO}_{2} / \mathrm{BiVO}_{4}$ interfaces, in which functional performance has been explained by invoking differing mechanisms, including hot carrier transfer, defect-mediated transport, and both Type I and Type II band alignment. These discrepancies arise from analyzing heterostructure behavior based on the properties of isolated component materials. However, the methodology and analysis presented here allows for identification of emergent phenomena when different materials are combined at the nanoscale. As such, this work highlights the importance of synergistic materials interactions in improving solar energy conversion efficiencies and provides insights that can aid in the design of functional nanocomposites.

\section{Acknowledgements}

This material is based upon work performed by the Joint Center for Artificial Photosynthesis, a DOE Energy Innovation Hub, supported through the Office of Science of the U.S. Department of Energy under Award Number DE-SC0004993 (nanocomposite fabrication, 
photoelectrochemical characterization), and by the Solar Photochemistry Program of the U.S. Department of Energy, Office of Science, Office of Basic Energy Sciences, Division of Chemical, Geological and Biosciences under Contract No. DE-AC02-05CH11231 (spectroscopy of semiconductors). L.H.H. acknowledges financial support from the Alexander von Humboldt Foundation. A.L acknowledges the H2020-Marie Curie Individual Fellowship with grant agreement number 701745 for financial support. R.B. thanks start-up funding from EPFL and the Swiss SNF (AP Energy Grant, project number PYAPP2_166897/1).

\section{References}

[1] Y. Tachibana, L. Vayssieres, J.R. Durrant, Artificial photosynthesis for solar watersplitting, Nat Photon, 6 (2012) 511-518.

[2] M.G. Walter, E.L. Warren, J.R. McKone, S.W. Boettcher, Q. Mi, E.A. Santori, N.S. Lewis, Solar Water Splitting Cells, Chemical Reviews, 110 (2010) 6446-6473.

[3] K. Sivula, R. van de Krol, Semiconducting materials for photoelectrochemical energy conversion, Nature Reviews Materials, 1 (2016) 15010.

[4] B. Kumar, M. Llorente, J. Froehlich, T. Dang, A. Sathrum, C.P. Kubiak, Photochemical and Photoelectrochemical Reduction of CO2, Annual Review of Physical Chemistry, 63 (2012) 541-569.

[5] Y. Park, K.J. McDonald, K.-S. Choi, Progress in bismuth vanadate photoanodes for use in solar water oxidation, Chemical Society Reviews, 42 (2013) 2321-2337.

[6] J.K. Cooper, S. Gul, F.M. Toma, L. Chen, P.-A. Glans, J. Guo, J.W. Ager, J. Yano, I.D. Sharp, Electronic Structure of Monoclinic BiVO4, Chemistry of Materials, 26 (2014) 53655373.

[7] J.K. Cooper, S. Gul, F.M. Toma, L. Chen, Y.-S. Liu, J. Guo, J.W. Ager, J. Yano, I.D. Sharp, Indirect Bandgap and Optical Properties of Monoclinic Bismuth Vanadate, The Journal of Physical Chemistry C, 119 (2015) 2969-2974.

[8] A.J.E. Rettie, H.C. Lee, L.G. Marshall, J.-F. Lin, C. Capan, J. Lindemuth, J.S. McCloy, J. Zhou, A.J. Bard, C.B. Mullins, Combined Charge Carrier Transport and Photoelectrochemical Characterization of BiVO4 Single Crystals: Intrinsic Behavior of a Complex Metal Oxide, Journal of the American Chemical Society, 135 (2013) 11389-11396.

[9] A.J.E. Rettie, W.D. Chemelewski, J. Lindemuth, J.S. McCloy, L.G. Marshall, J. Zhou, D. Emin, C.B. Mullins, Anisotropic small-polaron hopping in W:BiVO4 single crystals, Applied Physics Letters, 106 (2015) 022106.

[10] A.J.E. Rettie, W.D. Chemelewski, D. Emin, C.B. Mullins, Unravelling Small-Polaron Transport in Metal Oxide Photoelectrodes, The Journal of Physical Chemistry Letters, 7 (2016) 471-479.

[11] F.M. Toma, J.K. Cooper, V. Kunzelmann, M.T. McDowell, J. Yu, D.M. Larson, N.J. Borys, C. Abelyan, J.W. Beeman, K.M. Yu, J. Yang, L. Chen, M.R. Shaner, J. Spurgeon, F.A. Houle, K.A. Persson, I.D. Sharp, Mechanistic insights into chemical and photochemical transformations of bismuth vanadate photoanodes, Nat Commun, 7 (2016).

[12] X. Shi, I.Y. Choi, K. Zhang, J. Kwon, D.Y. Kim, J.K. Lee, S.H. Oh, J.K. Kim, J.H. Park, Efficient photoelectrochemical hydrogen production from bismuth vanadate-decorated tungsten trioxide helix nanostructures, Nat Commun, 5 (2014).

[13] A. Loiudice, J.K. Cooper, L.H. Hess, T.M. Mattox, I.D. Sharp, R. Buonsanti, Assembly and Photocarrier Dynamics of Heterostructured Nanocomposite Photoanodes from Multicomponent Colloidal Nanocrystals, Nano Letters, 15 (2015) 7347-7354.

[14] J. Resasco, H. Zhang, N. Kornienko, N. Becknell, H. Lee, J. Guo, A.L. Briseno, P. Yang, TiO2/BiVO4 Nanowire Heterostructure Photoanodes Based on Type II Band Alignment, ACS Central Science, 2 (2016) 80-88. 
[15] X. An, T. Li, B. Wen, J. Tang, Z. Hu, L.-M. Liu, J. Qu, C.P. Huang, H. Liu, New Insights into Defect-Mediated Heterostructures for Photoelectrochemical Water Splitting, Advanced Energy Materials, 6 (2016) n/a-n/a.

[16] H. Li, H. Yu, X. Quan, S. Chen, H. Zhao, Improved Photocatalytic Performance of Heterojunction by Controlling the Contact Facet: High Electron Transfer Capacity between $\mathrm{TiO} 2$ and the $\{110\}$ Facet of BiVO4 Caused by Suitable Energy Band Alignment, Advanced Functional Materials, 25 (2015) 3074-3080.

[17] J. Sun, X. Li, Q. Zhao, J. Ke, D. Zhang, Novel V2O5/BiVO4/TiO2 Nanocomposites with High Visible-Light-Induced Photocatalytic Activity for the Degradation of Toluene, The Journal of Physical Chemistry C, 118 (2014) 10113-10121.

[18] S. Ho-Kimura, S.J.A. Moniz, A.D. Handoko, J. Tang, Enhanced photoelectrochemical water splitting by nanostructured BiVO4-TiO2 composite electrodes, Journal of Materials Chemistry A, 2 (2014) 3948-3953.

[19] Y. Hu, D. Li, Y. Zheng, W. Chen, Y. He, Y. Shao, X. Fu, G. Xiao, BiVO4/TiO2 nanocrystalline heterostructure: A wide spectrum responsive photocatalyst towards the highly efficient decomposition of gaseous benzene, Applied Catalysis B: Environmental, 104 (2011) 30-36.

[20] M. Xie, X. Fu, L. Jing, P. Luan, Y. Feng, H. Fu, Long-Lived, Visible-Light-Excited Charge Carriers of TiO2/BiVO4 Nanocomposites and their Unexpected Photoactivity for Water Splitting, Advanced Energy Materials, 4 (2014) n/a-n/a.

[21] L. Zhang, G. Tan, S. Wei, H. Ren, A. Xia, Y. Luo, Microwave hydrothermal synthesis and photocatalytic properties of $\mathrm{TiO} 2 / \mathrm{BiVO} 4$ composite photocatalysts, Ceramics International, 39 (2013) 8597-8604.

[22] S. Obregon, G. Colon, A ternary Er3+-BiVO4/TiO2 complex heterostructure with excellent photocatalytic performance, RSC Advances, 4 (2014) 6920-6926.

[23] B.-Y. Cheng, J.-S. Yang, H.-W. Cho, J.-J. Wu, Fabrication of an Efficient BiVO4-TiO2 Heterojunction Photoanode for Photoelectrochemical Water Oxidation, ACS Applied Materials \& Interfaces, 8 (2016) 20032-20039.

[24] S.J.A. Moniz, S.A. Shevlin, D.J. Martin, Z.-X. Guo, J. Tang, Visible-light driven heterojunction photocatalysts for water splitting - a critical review, Energy \& Environmental Science, 8 (2015) 731-759.

[25] R. Buonsanti, E. Carlino, C. Giannini, D. Altamura, L. De Marco, R. Giannuzzi, M. Manca, G. Gigli, P.D. Cozzoli, Hyperbranched Anatase TiO2 Nanocrystals: Nonaqueous Synthesis, Growth Mechanism, and Exploitation in Dye-Sensitized Solar Cells, Journal of the American Chemical Society, 133 (2011) 19216-19239.

[26] H. Ye, J. Lee, J.S. Jang, A.J. Bard, Rapid Screening of BiVO4-Based Photocatalysts by Scanning Electrochemical Microscopy (SECM) and Studies of Their Photoelectrochemical Properties, The Journal of Physical Chemistry C, 114 (2010) 13322-13328.

[27] B. Iandolo, B. Wickman, I. Zoric, A. Hellman, The rise of hematite: origin and strategies to reduce the high onset potential for the oxygen evolution reaction, Journal of Materials Chemistry A, 3 (2015) 16896-16912.

[28] G.P. Nagabhushana, G. Nagaraju, G.T. Chandrappa, Synthesis of bismuth vanadate: its application in $\mathrm{H} 2$ evolution and sunlight-driven photodegradation, Journal of Materials Chemistry A, 1 (2013) 388-394.

[29] S.J. Hong, S. Lee, J.S. Jang, J.S. Lee, Heterojunction BiVO4/WO3 electrodes for enhanced photoactivity of water oxidation, Energy \& Environmental Science, 4 (2011) 17811787.

[30] S.P. Berglund, D.W. Flaherty, N.T. Hahn, A.J. Bard, C.B. Mullins, Photoelectrochemical Oxidation of Water Using Nanostructured BiVO4 Films, The Journal of Physical Chemistry C, 115 (2011) 3794-3802. 
[31] D.O. Scanlon, C.W. Dunnill, J. Buckeridge, S.A. Shevlin, A.J. Logsdail, S.M. Woodley, C.R.A. Catlow, M.J. Powell, R.G. Palgrave, I.P. Parkin, G.W. Watson, T.W. Keal, P. Sherwood, A. Walsh, A.A. Sokol, Band alignment of rutile and anatase TiO2, Nat Mater, 12 (2013) 798-801.

[32] U.I. Gaya, A.H. Abdullah, Heterogeneous photocatalytic degradation of organic contaminants over titanium dioxide: A review of fundamentals, progress and problems, Journal of Photochemistry and Photobiology C: Photochemistry Reviews, 9 (2008) 1-12.

[33] J. Disdier, J.-M. Herrmann, P. Pichat, Platinum/titanium dioxide catalysts. A photoconductivity study of electron transfer from the ultraviolet-illuminated support to the metal and of the influence of hydrogen, Journal of the Chemical Society, Faraday Transactions 1: Physical Chemistry in Condensed Phases, 79 (1983) 651-660.

[34] Y. Pihosh, I. Turkevych, K. Mawatari, J. Uemura, Y. Kazoe, S. Kosar, K. Makita, T. Sugaya, T. Matsui, D. Fujita, M. Tosa, M. Kondo, T. Kitamori, Photocatalytic generation of hydrogen by core-shell WO3/BiVO4 nanorods with ultimate water splitting efficiency, Scientific Reports, 5 (2015) 11141.

[35] W.-J. Yin, S.-H. Wei, M.M. Al-Jassim, J. Turner, Y. Yan, Doping properties of monoclinic BiVO $\left\{\{\}_{-}\{4\} \$\right.$ studied by first-principles density-functional theory, Physical Review B, 83 (2011) 155102.

[36] J.K. Cooper, S.B. Scott, Y. Ling, J. Yang, S. Hao, Y. Li, F.M. Toma, M. Stutzmann, K.V. Lakshmi, I.D. Sharp, The Role of Hydrogen in Defining the n-Type Character of BiVO4 Photoanodes, Chemistry of Materials, (2016).

[37] X. Pan, M.-Q. Yang, X. Fu, N. Zhang, Y.-J. Xu, Defective TiO2 with oxygen vacancies: synthesis, properties and photocatalytic applications, Nanoscale, 5 (2013) 3601-3614.

[38] W.N. Hansen, G.J. Hansen, Standard reference surfaces for work function measurements in air, Surface Science, 481 (2001) 172-184.

[39] E.A. Kraut, R.W. Grant, J.R. Waldrop, S.P. Kowalczyk, Semiconductor core-level to valence-band maximum binding-energy differences: Precise determination by $\mathrm{x}$-ray photoelectron spectroscopy, Physical Review B, 28 (1983) 1965-1977.

[40] R.W. Grant, E.A. Kraut, S.P. Kowalczyk, J.R. Waldrop, Measurement of potential at semiconductor interfaces by electron spectroscopy, Journal of Vacuum Science \& Technology B, 1 (1983) 320-327.

[41] J.R. Waldrop, S.P. Kowalczyk, R.W. Grant, E.A. Kraut, D.L. Miller, XPS measurement of GaAs-AlAs heterojunction band discontinuities: Growth sequence dependence, Journal of Vacuum Science \& Technology, 19 (1981) 573-575.

[42] L.-C. Tien, J.-L. Shih, Type-II [small alpha]-In2S3/In2O3 nanowire heterostructures: evidence of enhanced photo-induced charge separation efficiency, RSC Advances, 6 (2016) 12561-12570.

[43] Z. Zhang, C. Shao, X. Li, Y. Sun, M. Zhang, J. Mu, P. Zhang, Z. Guo, Y. Liu, Hierarchical assembly of ultrathin hexagonal $\mathrm{SnS} 2$ nanosheets onto electrospun TiO2 nanofibers: enhanced photocatalytic activity based on photoinduced interfacial charge transfer, Nanoscale, 5 (2013) 606-618.

[44] Z. Zhang, K. Liu, Z. Feng, Y. Bao, B. Dong, Hierarchical Sheet-on-Sheet ZnIn2S4/gC3N4 Heterostructure with Highly Efficient Photocatalytic $\mathrm{H} 2$ production Based on Photoinduced Interfacial Charge Transfer, Scientific Reports, 6 (2016) 19221.

[45] Y. Zheng, L. Zheng, Y. Zhan, X. Lin, Q. Zheng, K. Wei, Ag/ZnO Heterostructure Nanocrystals: Synthesis, Characterization, and Photocatalysis, Inorganic Chemistry, 46 (2007) 6980-6986.

[46] Z. Zhang, C. Shao, X. Li, C. Wang, M. Zhang, Y. Liu, Electrospun Nanofibers of p-Type $\mathrm{NiO} / \mathrm{n}$-Type $\mathrm{ZnO}$ Heterojunctions with Enhanced Photocatalytic Activity, ACS Applied Materials \& Interfaces, 2 (2010) 2915-2923. 
[47] F.J. Knorr, C.C. Mercado, J.L. McHale, Trap-State Distributions and Carrier Transport in Pure and Mixed-Phase TiO2: Influence of Contacting Solvent and Interphasial Electron Transfer, The Journal of Physical Chemistry C, 112 (2008) 12786-12794.

[48] F.J. Knorr, J.L. McHale, Spectroelectrochemical Photoluminescence of Trap States of Nanocrystalline TiO2 in Aqueous Media, The Journal of Physical Chemistry C, 117 (2013) 13654-13662.

[49] Y. Ma, S.R. Pendlebury, A. Reynal, F. Le Formal, J.R. Durrant, Dynamics of photogenerated holes in undoped $\mathrm{BiVO} 4$ photoanodes for solar water oxidation, Chemical Science, 5 (2014) 2964-2973. 


\section{Figures}
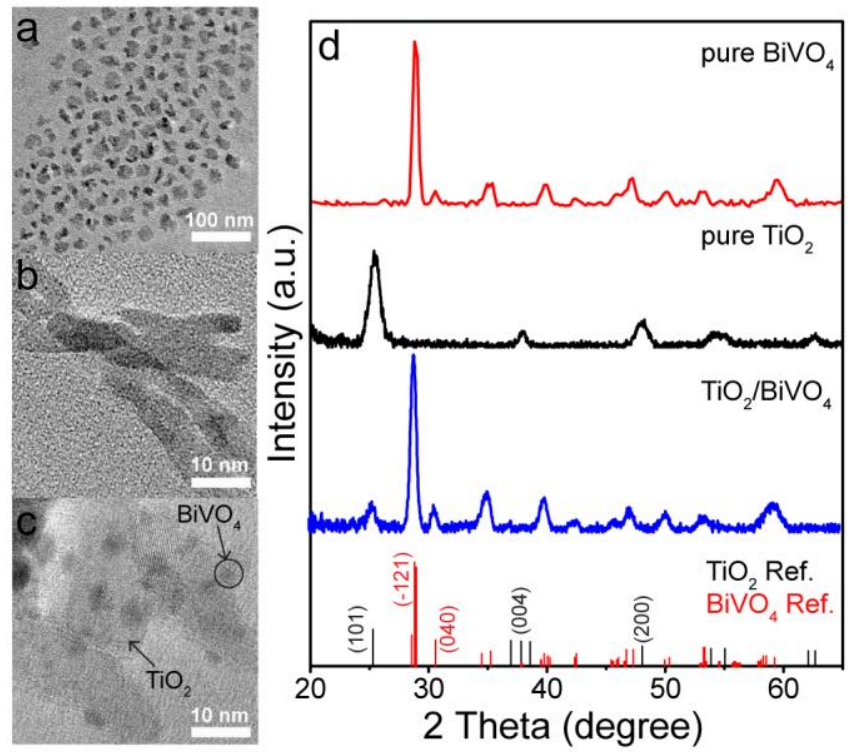

Figure 1. Transmission electron microscopy (TEM) images of (a) $\mathrm{Bi}_{2} \mathrm{O}_{2.7} / \mathrm{VO}_{\mathrm{x}}$ nanocrystal (NC) heterodimers, (b) $\mathrm{TiO}_{2}$ branched nanorods (NR), and (c) $\mathrm{TiO}_{2} / \mathrm{BiVO}_{4}$ nanocomposites. (d) X-ray diffractograms from pure $\mathrm{BiVO}_{4}$ nanocrystals (red), pure $\mathrm{TiO}_{2} \mathrm{NRs}$ (black), and $\mathrm{TiO}_{2} / \mathrm{BiVO}_{4}$ nanocomposites (blue). Also shown are the reference diffraction peak positions for pure phase anatase $\mathrm{TiO}_{2}$ (black) and monoclinic scheelite $\mathrm{BiVO}_{4}$ (red).
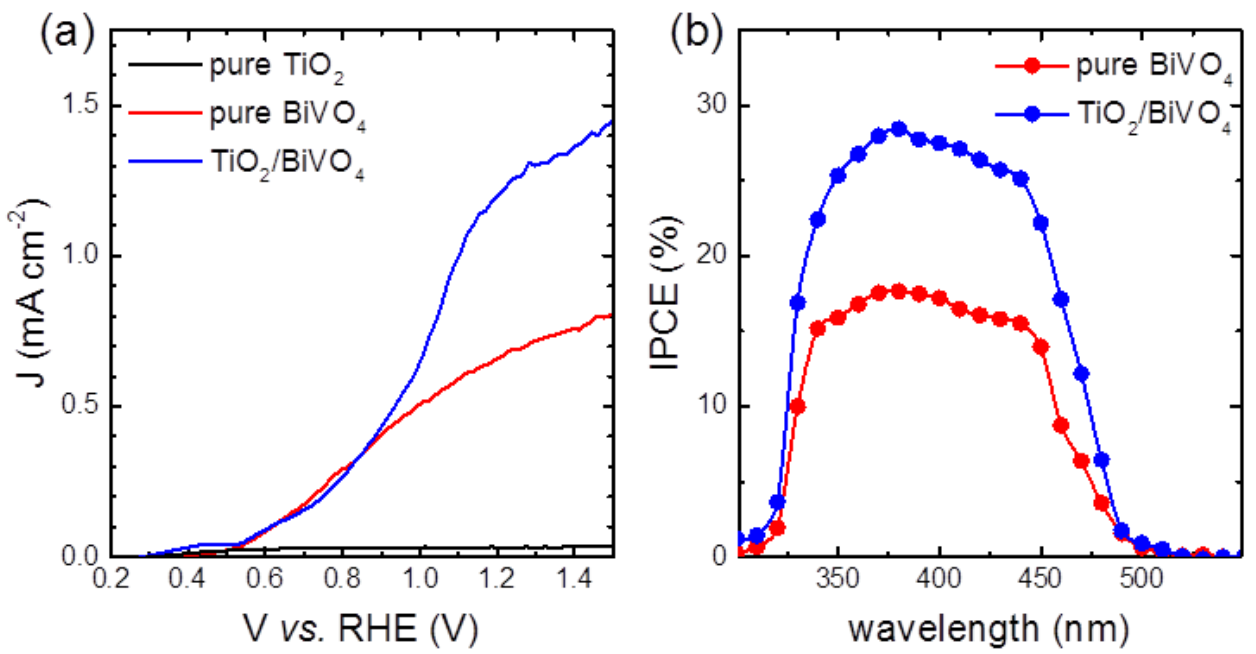

Figure 2. (a) Photocurrent density as a function of applied electrochemical potential for pure $\mathrm{TiO}_{2}$ (black), pure $\mathrm{BiVO}_{4}$ (red), and $\mathrm{TiO}_{2} / \mathrm{BiVO}_{4}$ nanocomposites (blue). (b) Incident-photonto-charge efficiency (IPCE) as a function of wavelength for pure $\mathrm{BiVO}_{4}$ (red) and $\mathrm{TiO}_{2} / \mathrm{BiVO}_{4}$ nanocomposites (blue). 

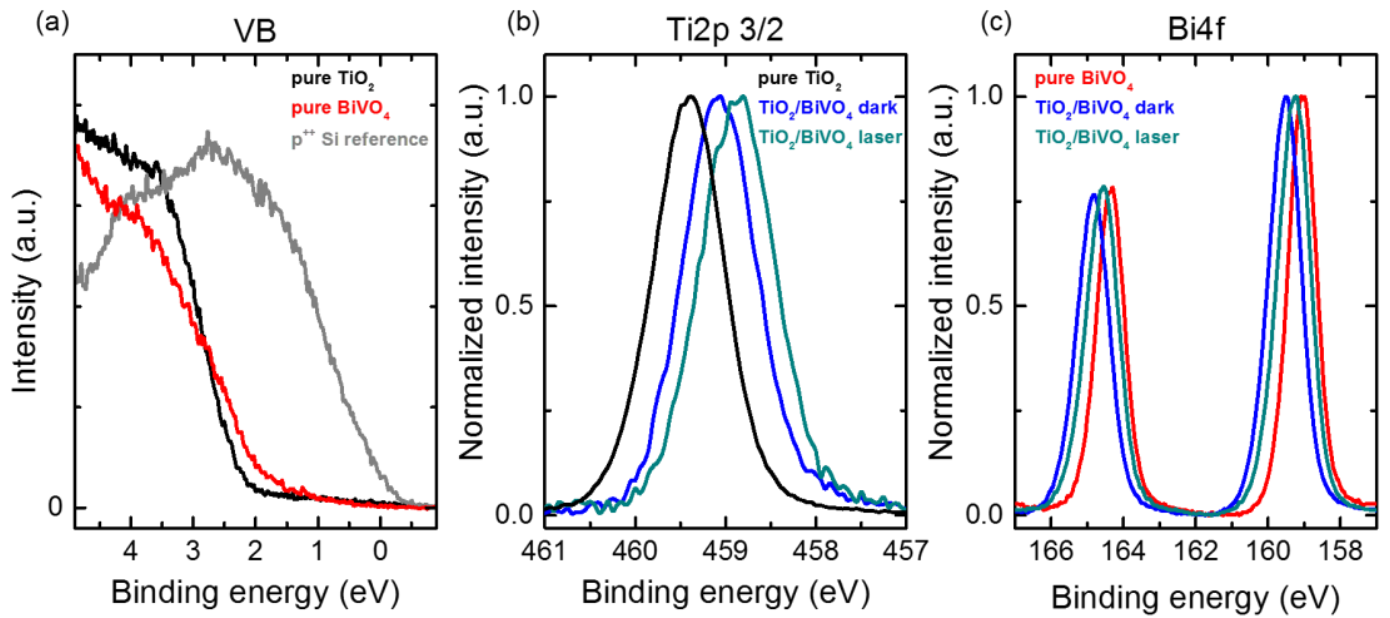

Figure 3. X-ray photoelectron spectra of (a) the valence band onsets for the pure $\mathrm{TiO}_{2}$ and $\mathrm{BiVO}_{4}$ films, as well as a $\mathrm{p}^{++} \mathrm{Si}$ reference sample, (b) the Ti $2 \mathrm{p}_{3 / 2}$ core level region in both pure $\mathrm{TiO}_{2}$ and the $\mathrm{TiO}_{2} / \mathrm{BiVO}_{4}$ nanocomposite in darkness and under illumination, and (c) the $\mathrm{Bi} 4 \mathrm{f}$ core level in pure $\mathrm{BiVO}_{4}$ and the $\mathrm{TiO}_{2} / \mathrm{BiVO}_{4}$ nanocomposite in darkness and under illumination. The spectra obtained under illumination in (b) and (c) were acquired with a 405 $\mathrm{nm}$ laser aligned to the XPS analysis location.

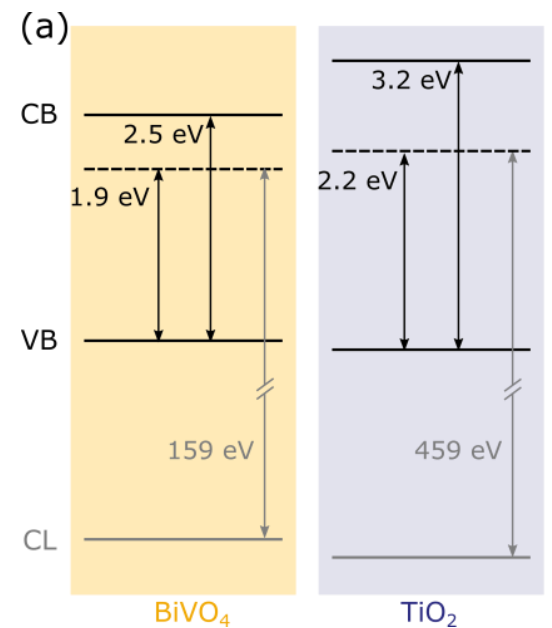

(b)

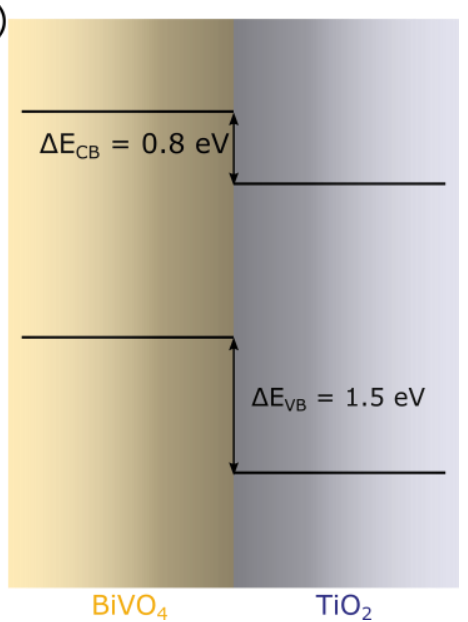

Figure 4. Interfacial band alignment. (a) Type I band alignment as predicted from pure phase measurements. (b) Type II band alignment obtained by photoemission measurements on the mixed nanocomposite. $\mathrm{CB}$ : conduction band, $\mathrm{VB}$ : valence band, $\mathrm{CL}$ : core level, $\Delta E_{C B}$ : conduction band offset, $\Delta E_{V B}$ : valence band offset. 


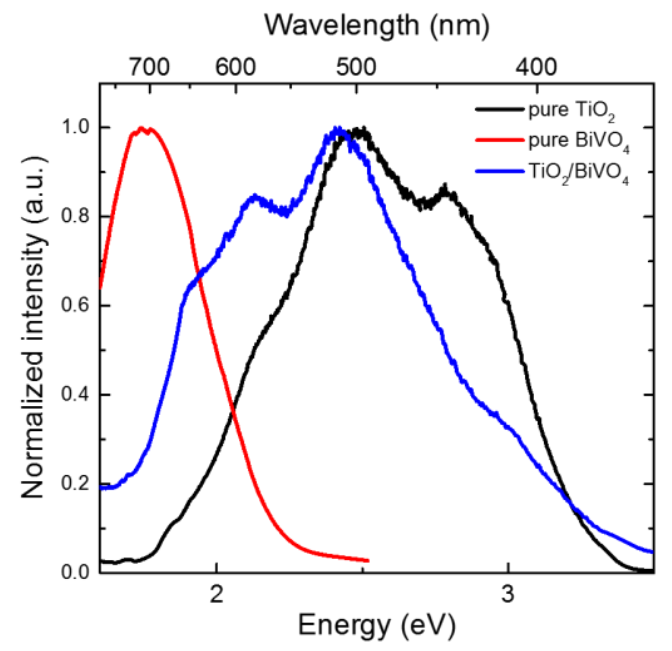

Figure 5. Normalized photoluminescence spectra measured on pure phase and mixed materials showing a suppression of bulk relative to surface recombination pathways relative in the nanocomposite (pump beam $\lambda=340 \mathrm{~nm}$ ).
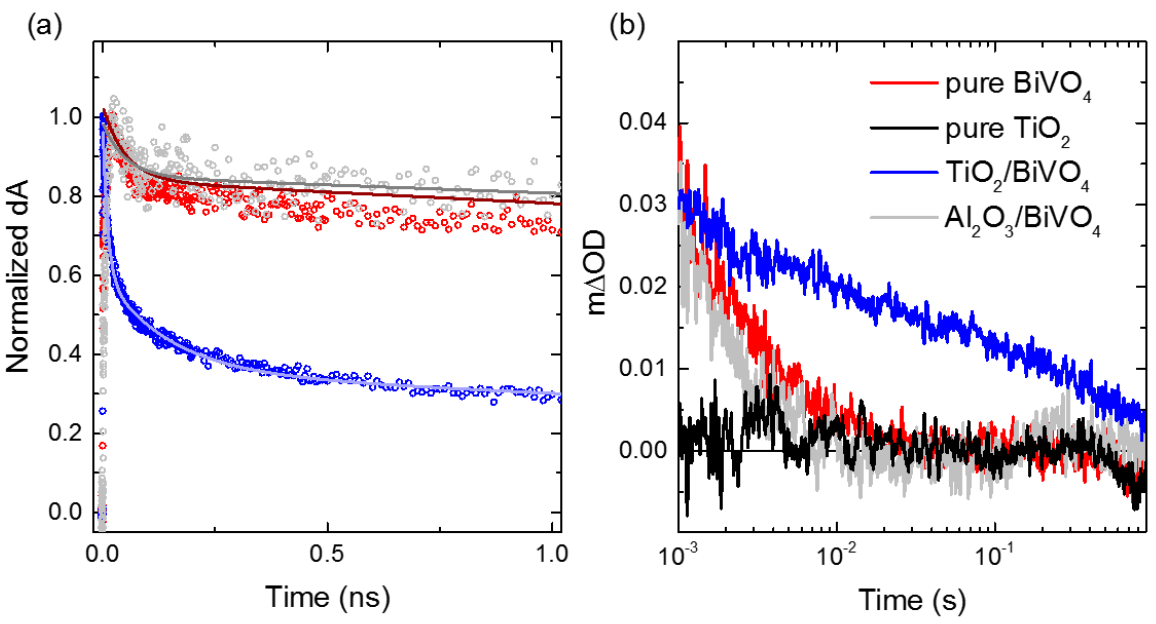

Figure 6. (a) Transient absorption decay response in the ps time scale (excitation pulse $\lambda=$ $350 \mathrm{~nm}$, transient signal probed at $475 \mathrm{~nm}$ ) of $\mathrm{TiO}_{2} / \mathrm{BiVO}_{4}$ and $\mathrm{Al}_{2} \mathrm{O}_{3} / \mathrm{BiVO}_{4}$ nanocomposites compared with the pure $\mathrm{BiVO}_{4}$ thin film. (b) Transient absorption decay traces of different nanocomposites in the $\mathrm{ms}$ time scale (excitation pulse $\lambda=355 \mathrm{~nm}$, transient signal probed at $550 \mathrm{~nm})$. 


\section{Vitae}
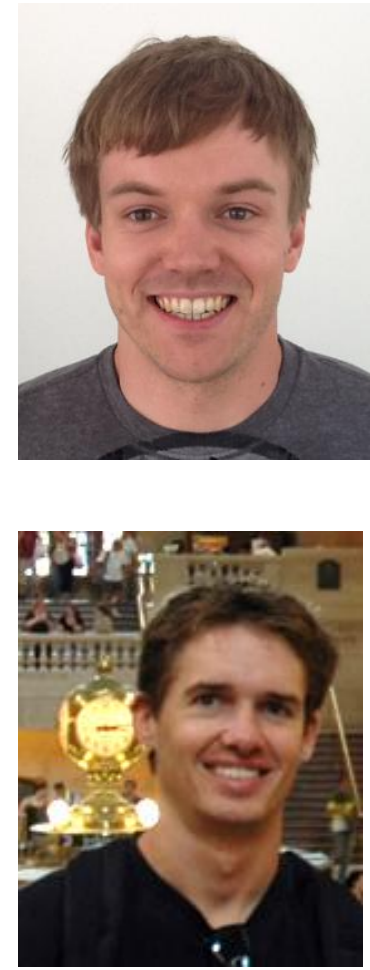

Lucas H. Hess received the "Diplomphysiker" degree from the Technische Universität München, Munich, Germany, in 2010, where he also obtained his Ph.D. degree in Physics in 2014 working on graphene biosensors at the Walter Schottky Institut. In 2014, Dr. Hess received a Feodor Lynen Research Fellowship by the Alexander von Humboldt foundation to work as a postdoctoral researcher at the Lawrence Berkeley National Laboratory. His current research focusses on understanding interfacial carrier recombination and charge transfer in photoelectrodes for solar energy conversion.

Jason K. Cooper is a Research Scientist at the Lawrence Berkeley National Laboratory, CA with the Joint Center for Artificial Photosynthesis (JCAP). He completed his B.S. in Chemistry from the California State University, Sacramento, CA and his $\mathrm{PhD}$ in Chemistry from the University of California, Santa Cruz, CA. He joined JCAP as a Postdoctoral Fellow in 2013. His research is principally focused on optical, electronic, and dynamic properties of semiconductor materials with an emphasis on x-ray, optical, and time resolved spectroscopies. Additionally, his research includes thin-film growth by sputtering, ALD, and spin-coating. His research efforts are aimed at understanding fundamental properties and defects of materials for applications in solar energy harvesting and conversion to chemical energy for storage.

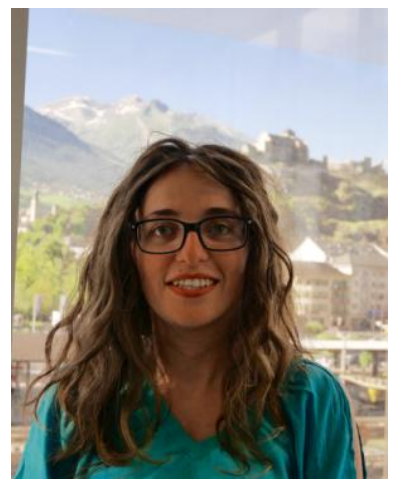

Anna Loiudice is a postdoctoral fellow at the Institute of Chemical Sciences and Engineering (ISIC) of the École Polytechnique Fédérale de Lausanne (EPFL) in the group of Prof. Raffaella Buonsanti. In 2014, after completing her master and $\mathrm{PhD}$ in Material Science at the University of Bari and at the Italian Institute of Technology in Lecce, respectively, she moved as a postdoctoral researcher at Lawrence Berkeley National Laboratory (LBNL), USA. In 2016 she has been awarded a Marie Curie Reintegration Fellowship to pursue her work on colloidal light absorbers for water splitting at EPFL. Her research interests span from nanomaterial synthesis to their integration in devices for energy applications.

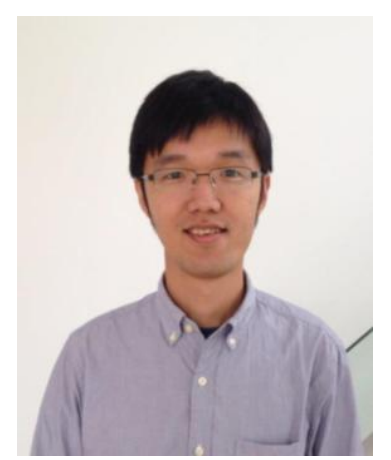

Chang-Ming Jiang is a postdoctoral researcher at the Joint Center for Artificial Photosynthesis (JCAP) in Lawrence Berkeley National Laboratory (LBNL). He received his $\mathrm{PhD}$ in Physical Chemistry from the University of California, Berkeley in 2015 before joined Dr. Ian D. Sharp's group at LBNL. His research interests include discovery and characterization of photoelectrode material for solar fuel generation, and utilizing ultrafast spectroscopic methods to study charge carrier dynamics in transition metal oxides. 


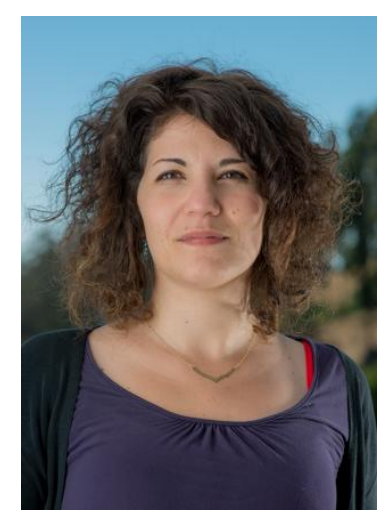

water oxidation.

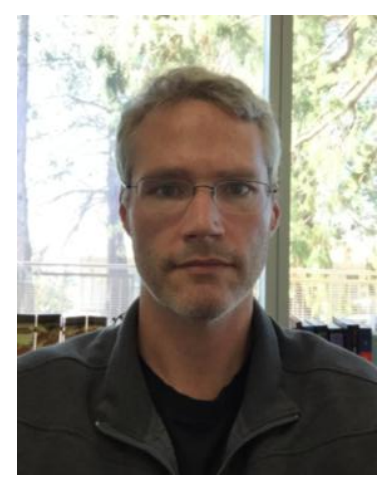

complex interfaces.
Raffaella Buonsanti is an Assistant Professor at at the Institute of Chemical Sciences and Engineering (ISIC) of the École

Polytechnique Fédérale de Lausanne (EPFL). After completing her master and $\mathrm{PhD}$ in Chemistry at the University of Bari and at the National Nanotechnology Laboratory, respectively, she moved as a postdoctoral researcher at Lawrence Berkeley National Laboratory (LBNL). From 2013 to 2015 Raffaella was a tenure-track Staff Scientist at LBNL, before moving to EPFL. Her group develops novel approaches, based on colloidal synthesis, to complex materials to drive chemical transformations, with particular emphasis on energy-related reactions such as $\mathrm{CO} 2$ reduction and
Ian Sharp is a Staff Scientist at the Lawrence Berkeley National Laboratory (LBNL) working in the Chemical Sciences Division and the Joint Center for Artificial Photosynthesis, where he is lead of the Materials Integration Thrust. He received a $\mathrm{PhD}$ in Materials Science and Engineering from UC Berkeley in 2006 and, from 2007-2011, was a researcher at the Walter Schottky Institut, Technische Universität München. His research is devoted to creating and understanding functional semiconductors for applications in renewable energy conversion, with a focus on electronic structure, dynamics, and chemical transformations at 
$\mathrm{BiVO}_{4} \mathrm{TiO}_{2}$

(a)

(9)

15

The

눈

ats 3

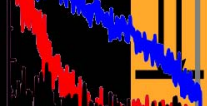

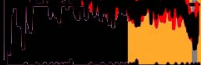

$b^{2}$

ase

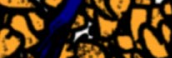

(3)

32

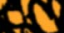

1.
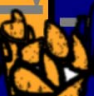

is

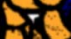

4

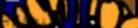

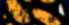

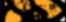

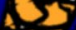

3

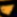

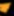

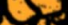

8

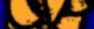

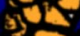

cor 